\title{
Le développement durable : stratégies descendantes et stratégies ascendantes
}

\author{
Sustainable development: \\ downwards and upwards strategies
}

\author{
Paul Claval \\ Université de Paris-Sorbonne \\ 29, rue du Soisy, 95600 Eaubonne
}

\section{Résumé}

Officialisée en 1987 par le Rapport Brundtland, l'idée de développement durable naît de la volonté de concilier défense des équilibres écologiques et amélioration des conditions de vie. Elle reçoit le soutien des grandes ONG écologiques. Les économistes, à la suite de Robert Solow, montrent que sous certaines conditions, impératifs écologiques et croissance sont conciliables. En 1992, la Conférence de Rio de Janeiro fait du développement durable une ardente obligation politique, à tous les niveaux. Par suite de l'urgence des problèmes que posent les transformations du climat, les objectifs sont déterminés au niveau global, puis répercutés à l'échelle des Etats. D'autres procédures, qui laissent plus d'initiatives au local ou au national, se combinent à la procédure descendante.

(C) 2006 Lavoisier, Paris. Tous droits réservés.

\section{Summary}

The idea of sustainable development, which was officialised by the Brundtland Report in 1987, was born from the will to reconcile the defence of ecological equilibria with the necessity to offer better conditions of life to the developing countries. It was supported from the start by the most important ecological NGOs. Following Solow, economists showed that, under specific conditions, economic growth was not conducive to environmental degradation. In 1992, the Rio de Janeiro Conference transformed sustainable growth into a political obligation for

\footnotetext{
*Adresse email : p.claval@wanadoo.fr

(C) 2006 Lavoisier, Paris. Tous droits réservés.
} 
political authorities, whatever their scale. Because of the urgency created by climatic change, targets were fixed at the top level, and then applied to every State. Other procedures, which give more initiative to the local and national levels, are also used.

(C) 2006 Lavoisier, Paris. Tous droits réservés.

Mots clés : Economie, développement, croissance, ressources, pollution, écosystème, écodéveloppement, capital naturel et capital technique, coordination internationale

Keywords: Economics, development, growth, resources, pollution, ecosystem, ecodevelopment, natural capital, technical capital, international cooperation

Née il y a un quart de siècle à peine, l'expression de développement durable a connu un succès fulgurant au cours des vingt dernières années. L'idée que la croissance doit constituer un des objectifs de la vie politique est récente : elle est née des réflexions de Keynes sur les origines de la Grande Crise, liée selon lui à l'incapacité des hommes d'affaires à anticiper les possibilités futures de l'économie, et à leur refus corrélatif de distribuer le pouvoir d'achat nécessaire pour que les productions nouvelles trouvent acquéreurs (Keynes, 1936). L'État doit donc se substituer à eux.

Les disciples de Keynes, Roy Harrod et Eusey Domar en particulier, montrent alors sur quoi doivent reposer les politiques macro-économiques indispensables à la croissance (Harrod, 1939 ; Domar, 1946/1957). Au lendemain de la Seconde Guerre mondiale, le progrès des comptabilités nationales révèle l'écart entre les pays riches et le reste du monde : il apparaît insupportable. Le discours que prononce le Président Truman en 1949, et où il parle pour la première fois de sous-développement, consacre la nouvelle direction de l'action politique au niveau national et au niveau international.

Les économistes découvrent que la croissance est instable et peut être dangereuse, car elle entraîne des difficultés - tensions inflationnistes ou déflationnistes en particulier. Pour assurer une croissance équilibrée (balanced growth), il convient de veiller à la fois sur le niveau des investissements et sur celui des moyens de paiement que ceux-ci permettent de créer. W. W. Rostow (1960) souligne alors que la dynamique de la croissance ne s'installe que progressivement : il parle du décollage vers la croissance durable (take-off into sustained growth) : le terme de durable est ainsi associé pour la première fois à celui de croissance.

Les premiers résultats des politiques menées sont décevants : il ne suffit pas de produire plus pour rendre les gens heureux. Ce dont les hommes rêvent, c'est d'un développement qui leur permette d'être en harmonie avec leur environnement et assure l'épanouissement de toutes leurs capacités.

La réflexion s'enrichit encore à la fin des années 1960 et au début des années 1970 : elle montre que la pauvreté est un mal social que les politiques conduites au nom de la croissance sont incapables de guérir. Les contraintes environnementales, jusque-là oubliées, sont désormais prises en compte : c'est le résultat de la publication du rapport Meadows en 1972 (Meadows et al., 1972) et le contre-coup du premier choc pétrolier survenu en 1973. C'est dans ce contexte que s'élabore la notion de développement durable. Elle résulte de la réflexion de spécialistes de l'atmosphère et du climat, de biologistes et d'économistes. La première partie de cette étude éclaire l'élaboration de la notion de développement durable des années 1970 à la Conférence de Rio de Janeiro en 1992. 
Celle-ci marque un tournant. La période de réflexion préalable est terminée. L'idée de développement durable échappe à ses initiateurs. Elle est adoptée par tout un chacun, par les chefs d'entreprises, par les hommes politiques, par les publicistes. Elle est mise en œuvre à tous les échelles, du global au local. Elle était née du travail de recherche et des réflexions de spécialistes des sciences dures, de biologistes et d'économistes. Maintenant qu'elle navigue en eaux libres, elle devient une des facettes de la réalité concrète : comme telle, elle appelle les investigations des géographes. Ces derniers s'attachent en particulier aux stratégies descendantes et ascendantes qu'elle implique, et aux ajustements d'échelle qu'elle nécessite. C'est à ces aspects du développement durable qu'est consacrée la deuxième partie de cette étude.

\section{L'idée de développement durable : origine et raisons d'un succès}

\subsection{L'idée de développement durable}

La Conférence mondiale des Nations unies sur l'Environnement qui s'est tenue à Stockholm en 1972, avait prévu la mise en place de la « Commission mondiale sur l'environnement et le développement ». Celle-ci a commencé ses travaux en 1984. Présidée par le Premier Ministre norvégien, Mme Gro Harlem Brundtland, elle a présenté en 1987, un rapport : "Our Common Future », «Notre avenir à tous »(CMEE, 1988). Il résume par une formule simple la réflexion alors menée sur les conditions du développement : au lieu de chercher à maximiser ses activités économiques, l'humanité doit s'orienter vers le développement durable. L'expression, qui connaît dès lors un immense succès, était apparue sept ans plus tôt dans le sous-titre d'une étude préparée par l'Union Internationale pour la Conservation de la Nature, l'UICN : Stratégie mondiale de la croissance La conservation des espèces vivantes au service du développement durable (UICN/PNUE/WWF, 1980).

La montée des préoccupations écologiques était sensible depuis la fin des années 1950 (Dorst, 1965). La Conférence de Stockholm avait proposé, en 1972, de mettre la sauvegarde de l'environnement au premier plan des préoccupations des Nations Unies : pour y parvenir, il fallait, selon les naturalistes, renoncer à toute forme de croissance. Les classes défavorisées des pays riches et les populations du Tiers Monde ne pouvaient accepter une orientation qui les condamnerait à la médiocrité. Maurice Strong, le grand fonctionnaire canadien des Nations Unies qui a orchestré toute l'action dans le domaine de la croissance des années 1960 aux années 1990, en était profondément conscient : en 1971, à l'occasion d'un colloque sur l'environnement organisé à Founex, en Suisse, il fait part à Serge Antoine, un des responsables de la politique française d'aménagement, des questions que pose déjà pour lui la Conférence de Stockholm qu'il prépare : «Au fait, c'est une conférence sur l'environnement, mais le développement là-dedans ? Comment ces deux notions peuvent-elles travailler ensemble ?» (Antoine, in Smouts, 2005, p. 84).

La Commission Brundtland propose une solution : elle reconnaît aux hommes le droit au développement, mais leur demande de le mener d'une telle manière qu'il ne compromette pas le futur de la planète :

« Deux concepts sont inhérents à la notion [de développement durable] : le concept de besoin, et plus particulièrement des besoins essentiels des plus démunis auxquels il convient d'accorder la plus grande priorité, et l'idée des limitations que l'état de 
nos techniques et de notre organisation impose à la capacité de l'environnement à répondre aux besoins actuels et à venir »(CMEE, 1988, Notre Avenir à tous, Montréal, Éditions du Fleuve, p. 51).

On comprend donc la nature ambiguë de la notion de développement durable : ce n'est pas un concept qui décrirait une réalité existante; c'est une injonction faite aux hommes pour, que sans renoncer à la satisfaction de leurs besoins, ils laissent aux générations futures un environnement qui leur soit aussi - ou plus - propice que celui que nous connaissons.

La formule reconnaît aux hommes le droit d'accéder un jour à des niveaux de consommation et de bien-être qui les font rêver ; elle leur impose de respecter l'environnement. Il y a une contradiction entre les deux termes associés, comme Gilbert Rist (1996), le souligne fortement : si l'on suit l'exemple des pays industriels, le développement est tout sauf durable ! C'est cette ambiguïté qui assure le succès de la formule, mais rend sa mise en ouvre délicate : comment assurer le développement sans accentuer les déséquilibres qui menacent la nature?

La formule est officialisée par la Conférence de Rio de Janeiro en 1992. Elle définit dès lors les buts que l'action de l'humanité doit s'assigner. Elle est instantanément adoptée par les aménageurs, les hommes politiques, les chercheurs et le grand public. C'est comme cela que sont aujourd'hui analysés les rapports entre les hommes, l'espace et la nature.

\subsection{Une conception parmi d'autres des relations des groupes humains à l'environnement}

D'autres formules ont été tour à tour proposées pour aborder ce problème : dans l'optique des Lumières du XVIII ${ }^{e}$ siècle et dans celle des philosophies de la nature du début du XIX ${ }^{\mathrm{e}}$, la nature est un dispositif mis en place par Dieu pour servir de cadre à la vie des hommes : l'harmonie des paysages naturels traduit la bénévolence du Créateur ; étudier le milieu, c'est analyser les formes physiques et vivantes qui nous environnent pour voir à quel dessein elles obéissent (sur l'histoire des conceptions de la nature et de l'écologie : Bourg, 1993 ; Cadoret, 1985 ; Deléage, 1991 ; Hayward, 1994 ; Milton, 1996 ; Pepper, 1996).

La montée de l'évolutionnisme change la signification de l'environnement : celui-ci n'est plus conçu comme un cadre destiné à l'épanouissement équilibré de la vie ; il est tissé de flux d'énergie et de matière qui permettent aux plantes et aux animaux de vivre et de se reproduire - mais cette nature est avare, si bien que la concurrence est dure entre les êtres ; les plus faibles sont éliminés ; c'est le moteur de la sélection naturelle et donc de l'évolution. Celle-ci confère à la nature son dynamisme : elle donne naissance à de nouvelles formes de vie, supérieures aux précédentes.

L'environnementalisme darwinien est vite jugé excessif lorsqu'il est appliqué aux groupes sociaux : dans une optique néo-lamarckienne, l'évolution de l'homme est plutôt vue comme un processus d'adaptation que comme le résultat d'une sélection aveugle. L'humanité innove et apprend ainsi à mieux tirer partie de l'environnement : c'est l'optique possibiliste.

Il faut attendre l'entre-deux-guerres pour que les bases de l'écologie s'affermissent : c'est alors que commence vraiment l'analyse des chaînes trophiques qui alimentent les êtres vivants et assurent la survie des espèces. Ces chaînes font partie de systèmes : les 
écosystèmes. L'idée de prendre en compte la dimension spatiale de ceux-ci s'impose dans les années 1960 : on se met à parler de géosystèmes (Rougerie et Beroutchachvili, 1991). Ceux-ci se traduisent dans les formes visibles, dans les paysages ; ces derniers sont ainsi au centre d'une des conceptions les plus fécondes des rapports entre hommes et milieux.

L'idée de développement durable permet de formuler d'une autre manière les rapports entre sociétés, cultures et nature. Pour bien le comprendre, il faut analyser l'évolution des relations nouées avec l'environnement en fonction des niveaux techniques.

\subsection{Développement durable et révolutions écologiques}

Dans les sociétés primitives aussi bien que dans les sociétés traditionnelles - et pour une grande partie de l'espace terrestre, jusqu'au milieu du $\mathrm{XX}^{\mathrm{e}}$ siècle encore -, les groupes dépendaient en totalité de l'énergie solaire transformée par les plantes en énergie chimique potentielle, celle des aliments ou du bois de chauffage, ou véhiculée sous forme d'énergie mécanique par les turbulences de l'air et les mouvements des eaux. Les hommes n'avaient pas accès à des formes concentrées d'énergie : pour eux, la nature était avare. C'est la vision qui domine au moment où l'environnementalisme triomphe.

La révolution industrielle modifie la situation : la mobilisation des énergies fossiles et la meilleure utilisation de celles du vent et des eaux permettent de mettre en œuvre des formes concentrées d'énergie en quelques points ou régions ; elles conduisent aussi à l'amélioration des transports : une bonne partie des contraintes que l'environnement imposait aux hommes disparaît maintenant que ceux-ci peuvent s'approvisionner ailleurs. Comme les formes d'énergie concentrées ne sont encore disponibles qu'en un petit nombre de points, il est facile d'exporter les déchets et les eaux usées qu'elles produisent vers des zones à utilisation moins intensive, où ils sont recyclés par les écosystèmes en place. La première révolution écologique a eu lieu : elle conduit à une vision optimiste des rapports entre les hommes et leur environnement.

La poursuite du progrès modifie cette situation. Il est désormais possible d'avoir partout accès à des formes concentrées d'énergie, si bien que la solution qui consiste à exporter les déchets vers des zones encore traditionnelles n'est plus possible. Les écosystèmes touchés par la modernisation ne sont pas capables de traiter les quantités croissantes de matière qui leur sont injectées. Leur limite de résilience a été dépassée. L'environnement se dégrade. Le temps est de nouveau au pessimisme écologique, mais cette fois, ce n'est plus la nature qui en est coupable par suite de son avarice. Ce sont les hommes qui en sont responsables, parce qu'ils détraquent les systèmes naturels. La recherche du développement durable correspond exactement à cette situation : les hommes sont désormais comptables du devenir de la nature. C'est la seconde révolution écologique du monde contemporain. Celle-ci modifie la place que tient l'humanité dans l'environnement, et lui impose la prévoyance en ce domaine (Jonas, 1990). Le principe de responsabilité s'impose :

«Le principe responsabilité prend acte d'un changement majeur : l'essence de l'agir humain s'est transformée. Les hommes ont désormais les moyens techniques d'altérer puis de détruire de manière irréversible et définitive leur "environnement" - ce dernier terme remplaçant d'ailleurs significativement le mot “Nature " qu'il n'est sans doute plus pertinent d'employer» (Cobast, 2005, p. 23-24). 
La Commission Brundtland est très consciente des dangers que l'évolution récente fait naître et de la nécessité d'adopter, pour les traiter, une attitude plus solidaire. Parmi les dangers qui la conduisent à formuler ses recommandations, trois jouent un rôle décisif parce qu'ils risquent d'entrâner des perturbations irréversibles au niveau mondial: (i) l'accumulation des gaz à effet de serre et (ii) les graves atteintes à la couche d'ozone provenant des produits fluoro-chlorés ; (iii) la menace d'extinction de nombreuses espèces, car elle remet en cause la biodiversité de la planète. Les deux premiers facteurs sont responsables du changement climatique.

\subsection{A l'arrière-plan des transformations : de nouvelles conceptions de la nature}

L'idée que l'environnement est menacé ne date pas des années 1950 ou 1960. Elle s'appuie sur une conviction : avant que l'homme ne parte à la conquête de la planète, un ordre harmonieux y existait. La végétation y prenait la forme de climax stables. Cet équilibre permettait à des centaines de milliers d'espèces de plantes et d'animaux de coexister. Il se traduisait par la beauté de paysages. L'idéal qui anime les conservationnistes est souvent aristocratique (il convient de sauvegarder des ensembles que l'activité des classes moyennes ou modestes menace) ; il a également une dimension pratique : il faut éviter le gaspillage de ressources précieuses. Des associations se consacrent à ces actions de protection. Elles essaient d'obtenir des hommes politiques qu'ils créent des réserves et des parcs. La plus célèbre d'entre elle est le WWF, World Wildlife Fund ou Fonds Mondial pour la Nature.

La deuxième révolution écologique transforme les données : le problème de la conservation change d'échelle : il ne suffit plus de mettre en défends des zones plus ou moins étendues afin de sauvegarder des morceaux de vraie nature. Il s'agit d'intervenir en un nombre croissant de points, et sur des étendues de plus en plus vastes, car les déséquilibres s'y multiplient. Ils deviennent globaux : on connaissait le rôle de l'effet de serre dans l'équilibre thermique de la terre depuis les travaux de Sven Arrhenius à la fin du XIX siècle. Le recours croissant aux énergies fossiles augmente l'émission de gaz à effet de serre : cela risque de provoquer un réchauffement climatique, cependant que l'utilisation des fréons - les composés fluoro-chlorés - menace la continuité de la couche d'ozone, qui protège la terre du rayonnement ultra-violet (sur l'apport de la physique de l'atmosphère et de l'écologie à l'analyse de ces transformations : Allègre, 1990 ; Larrère et Larrère, 1997 ; Le Treut et Janovici, 2004). Face à ces nouveaux défis, de nouveaux mouvements écologiques voient le jour dans les années 1960 : dans le monde anglo-saxon, les deux plus représentatifs sont Greenpeace et Friends of the Earth. Ces mouvements attirent des milliers de naturalistes et de chercheurs. C'est au sein de ces organisations non gouvernementales $(\mathrm{ONG})$ que mûrit la réflexion sur l'équilibre entre nature et sociétés.

La deuxième révolution écologique déplace les objectifs des groupes écologiques : créer des parcs ne suffit plus, à l'heure où d'immenses zones sont menacées par la pollution qu'engendrent villes et concentrations industrielles, et où l'on prend conscience de l'effet de serre. L'image traditionnelle de la nature s'efface. Les systèmes écologiques vont de crise en crise (Pickett et White, 1985). Ils ne fonctionnent pas à l'équilibre (Lepart, 1997). L'objectif ne peut plus être de revenir à un état idéal qui n'a sans doute jamais existé. Ce qui compte désormais, c'est de préserver la biodiversité. La notion était familière aux écologistes (Barbault, 1997 ; Blondel, 2005 ; Guillaud, 2005 ; Huston, 1994 ; Le Prestre, 2002 ; 
Myers et al., 2000 ; Vivien, 2005). Elle prend une valeur politique : on ne se bat plus pour conserver de beaux boisements. On essaie de prévenir une nouvelle grande extinction de la faune, comme l'histoire géologique en a connu plusieurs (Ramade, 1999).

La biodiversité s'exprime à trois niveaux : (i) celui de la diversité des espèces, le plus connu, celui pour lequel on dispose du plus grand nombre de travaux ; (ii) celui de la diversité des écosystèmes, pris surtout en compte à partir des années 1960 ; (iii) celui de la diversité des gènes, que les travaux sur l'ADN permettent de mieux comprendre.

La biodiversité paraît d'autant plus importante à préserver qu'elle assume un quadruple rôle : (i) elle est garante de l'approvisionnement alimentaire des êtres humains ; (ii) elle joue un rôle essentiel dans la régulation des écosystèmes, qui sont d'autant moins fragiles que la vie y revêt des formes plus variées ; (iii) elle constitue le support sur lequel les écosystèmes sont bâtis ; (iv) elle fait partie du patrimoine de l'humanité, dans la mesure où elle contribue au bien-être des hommes, prend pour eux une dimension esthétique, et garantit, grâce à l'utilisation de certaines espèces, leur développement économique.

Les personnages-clefs dans ces mouvements changent aussi : au naturaliste d'hier se substitue le biologiste. Derrière lui se profile le chimiste. Ce qui compte, au-delà de la multiplicité des espèces, c'est la diversité des gènes et la complexité des molécules qu'ils permettent d'élaborer. L'essor des biotechnologies renforce cette évolution. Pour découvrir les nouvelles molécules dont elles ont besoin pour créer des remèdes plus efficaces, les grandes firmes pharmaceutiques demandent à des ethnobotanistes de les renseigner sur les connaissances que les populations primitives ont acquises des propriétés thérapeutiques des espèces végétales au milieu desquelles elles vivent.

Les philosophes s'interrogent sur la signification de ces transformations (Bourg, 1996 ; Ferry, 1992 ; Jonas, 1990).

\subsection{A l'arrière-plan des transformations : les approches sociales de la crise environnementale}

La transformation des conceptions que l'on se fait de la nature bouleverse l'interprétation que l'on donne des menaces qui pèsent sur l'environnement. Dans l'optique où étaient nés les mouvements environnementalistes de la fin du XIX ${ }^{\mathrm{e}}$ siècle et de la première moitié du $\mathrm{XX}^{\mathrm{e}}$, c'étaient les populations paysannes, ou les humanités primitives, qui menaçaient les équilibres naturels. Le verdict reposait sur un fond malthusien : les familles trop nombreuses des populations paysannes obligeaient les jeunes à défricher de nouveaux espaces, réduisant ainsi les étendues boisées. Les élites urbanisées avaient accès aux certitudes de la science moderne; elles n'avaient que mépris à l'égard des pratiques de ceux qui ne pouvaient mobiliser que de vieux savoirs vernaculaires : les pasteurs étaient sévèrement jugés à cause des feux qu'ils multipliaient pour étendre leurs pâturages et offrir de jeunes pousses à la dent de leurs troupeaux.

Dans des pays vides comme l'Ouest des Etats-Unis, la création de parcs naturels où défrichements et feux étaient interdits ne menaçait pas l'équilibre des populations traditionnelles. En Afrique, il en allait autrement : les grands parcs que le colonisateur multiplie en Afrique du Sud et en Afrique de l'Est privent des ethnies entières de leurs territoires de chasse ou de pâturages pour leurs troupeaux. Ces politiques survivent à la fin de la colonisation - elles ont l'avantage de susciter des courants touristiques importants, et de générer des 
devises dans des pays qui en manquent cruellement. Sylvie Brunel le rappelle fortement : « [on] s'attache d'abord à "mettre l'Afrique sous cloche" en la transformant en une sorte de conservatoire de la faune et de la flore qui fait bien peu de cas des besoins des populations. Le monde développé assigne ainsi au continent noir la vocation d'une immense réserve exotique à visée récréative pour des Blancs en mal de nature sauvage et préservée » (Brunel, 2005, p. 351).

\section{D'où son cri :}

«La priorité en Afrique doit être donnée aux hommes - à leurs besoins non satisfaits, dans les domaines de l'éducation, de la santé et des infrastructures particulièrement, et à leurs conditions de vie trop difficiles et trop incertaines pour leur permettre d'abandonner les stratégies de survie et d'avoir confiance en l'avenir » (Brunel, 2005, p. 352).

Pour sauvegarder la nature dans les pays du Tiers Monde (l'expression a encore cours dans les années 1970 et 1980), il importe donc d'abord d'y lutter contre la pauvreté en y répandant l'instruction, en y améliorant la santé, en y créant des emplois (Blaikie et Brookfield, 1987 ; Martinez-Alier, 2002).

Pour les analystes d'aujourd'hui, ce ne sont plus les populations attardées - les masses paysannes des pays industrialisées (elles achèvent alors de disparaître) ou les populations des pays en voie de développement - qui sont les grandes responsables des atteintes contre l'environnement. C'est le monde industrialisé : c'est parce que ses industries consomment des quantités colossales de charbon, de pétrole, de gaz ou d'électricité de toute origine, et parce que ses populations consomment de plus en plus d'énergie pour chauffer ou climatiser leurs maisons ou pour se déplacer, que les rejets de gaz à effet de serre se multiplient, et que l'équilibre global de la planète se modifie.

Pour préserver la nature dans les pays en voie de développement, il convient d'abord d'y arracher les plus pauvres à la misère. Dans les pays riches, il faut persuader les consommateurs et les firmes de se montrer moins gourmands d'énergie et de matières premières.

\subsection{A l'arrière-plan des transformations : l'évolution de la pensée économique}

Le mouvement environnementaliste des années 1970 et 1980 aurait donc dû déboucher sur une remise en cause du développement, ou du moins des formes qu'il a prises dans les pays d'économie libérale ou dans ceux qui ont adopté les recettes de l'économie soviétique. Pour ceux qui conçoivent ainsi la sauvegarde de la nature - et partant, de l'humanité -, une réduction drastique des consommations s'imposerait. En la caricaturant un peu, on peut résumer ainsi leur attitude :

«Dans une économie saine, l'énergie fossile disparaîtrait. Elle serait réservée à des usages de survie comme les usages médicaux. Le transport aérien, les véhicules à moteur à explosion seraient condamnés à disparaître. Ils seraient remplacés par la marine à voile, le vélo, le train, la traction animale [...]. Bien entendu, toute notre civilisation serait bouleversée par ce changement par rapport à l'énergie. Il signifierait la fin des grandes surfaces au profit des commerces de proximité et des marchés, des produits manufacturés peu chers importés au profit d'objets produits localement, des emballages jetables au profit des contenants réutilisables, de l'agriculture intensive 
motorisée au profit d'une agriculture paysanne. Le réfrigérateur serait remplacé par une pièce froide, le voyage aux Antilles par une randonnée à vélo dans les Cévennes, l'aspirateur par le balai et la serpillière, l'alimentation carnée par une nourriture quasi végétarienne, etc. » («Altermonde le Village », cité par Mayeur, 2005, p.85-86).

Les tenants de l'écologisme débouchent ainsi sur une écopolitique très critique (Passet, 1996 ; Robbins, 2004). Ils voudraient initier un cycle de décroissance économique pour diminuer les pressions sur l'environnement (Georgescu-Roegen, 1895 ; Grinevald, 2002).

Au début des années 1970, certains chercheurs se demandent si des solutions aussi radicales s'imposent. Ils essaient de concilier sauvegarde de l'environnement et croissance modérée - ils prônent l'écodéveloppement ; le terme a sans doute été inventé par Maurice Strong ; Ignaczy Sachs s'en fait le théoricien (Sachs, 1980 ; voir aussi Illich, 1973 ; Bourg, 1995 ; Harribey, 1997 ; Le Prestre, 1997 ; Maréchal et Queneault, 2005).

Au pessimisme radical de ceux qui désirent imposer des limitations sévères à la croissance pour stopper la dégradation planétaire de l'environnement, et à la sagesse de ceux qui essaient de concevoir un écodéveloppement très prudent, s'oppose un courant beaucoup plus optimiste (Coase, 1960 ; Costanza et al., 1997 ; Grossman et Krueger, 1995 ; Hartwick, 1977 ; Hunt, 1989) ; il s'inspire de l'économie néo-classique. Lorsqu'ils procèdent à des échanges marchands, les hommes considèrent comme équivalents des biens qui ne se ressemblent pas et qui n'ont pas les mêmes usages : on parle de leur «marchandisation » ou de leur «commodification». Il est ainsi possible de les vendre et de les acheter : on sait depuis Adam Smith que cela conduit à la spécialisation du travail et au progrès.

La réflexion sur les équivalences entre biens peut aussi s'étendre aux biens capitaux. Ceux-ci sont de nature variée : capital naturel que celui que représentent la fertilité des terres ou les ressources minérales, capital humain que celui qui résulte de l'accumulation des savoirs et des compétences, capital technique que celui qui prend la forme de machines et d'investissements matériels.

Le capital naturel existe sous deux formes : renouvelable ou épuisable. Tout le monde s'accorde à considérer que le développement ne menace pas les équilibres naturels tant qu'il ne mobilise que des ressources renouvelables. Les économistes néo-classiques qui s'inspirent des recherches de Robert M. Solow estiment qu'il est aujourd'hui possible de substituer des formes de capital humain ou technique au capital naturel que le progrès détruit (Solow, $1972 ; 1977 ; 1999$ ).

Une constatation étaye cet optimisme raisonnable : lorsque le revenu s'élève, « les émissions polluantes augmentent jusqu'à une certaine limite, puis décroissent » (Boisvert et Vivien, 2006, p. 34). L'évolution des technologies accentue ce phénomène : on peut mettre au point, grâce à l'électronique, des équipements qui utilisent de manière plus rationnelle l'énergie et les matières premières. Les goûts des consommateurs évoluent aussi : leur demande se porte davantage vers les services, ce qui n'implique pas de consommation aussi élevée d'énergie et de matière.

Pour les néo-libéraux, le progrès permet donc de remplacer le capital naturel par des formes de capital générées par l'activité humaine.

«L'hypothèse retenue par les théoriciens néo-classiques est celle de la substituabi-

lité entre les différentes formes de capital : une quantité accrue de "capital créé par 
les hommes" (le stock d'équipements, de connaissances, d'équipments, le niveau général d'éducation et de formation) doit prendre le relais de quantités moindres de "capital naturel" (le stock de ressources naturelles disponibles), pour assurer le maintien, à travers le temps, des capacités de production et de bien-être des individus » (Boisvert et Vivien, 2006, p. 33-34).

Pour que le progrès se poursuive ainsi, il suffit d'investir « les rentes procurées par l'exploitation des ressources naturelles dans le capital technique » (Boisvert et Vivien, 2006, p. 34).

Marie-Claude Smouts résume ainsi les nouvelles théories de la croissance durable :

«Pour toute une école de l'économie de l'environnement, il suffit que, selon la règle de Hartwick, les rentes issues de l'exploitation des ressources naturelles soient réinvesties sous la forme d'un capital reproductible qui se substituera aux actifs naturels. Si ces actifs reproductibles sont transmis aux générations futures dans des proportions qui permettent de maintenir les niveaux de consommation réels au cours du temps, l'objectif de soutenabilité et d'équité intergénérationnelle sera rempli (règle de Hicks, Hartwick, Solow). Dans cette logique de “soutenabilité faible', la dégradation de l'environnement est considérée comme parfaitement acceptable dès lors que des investissements compensatoires ont été effectués » (Smouts, 2005, p. 8-9).

Une philosophie se dégage des travaux de ces économistes :

« [Leur] analyse renvoie, dans une acception schumpetérienne, à une sorte de création destructrice à vocation écologique qui voit la mise en œuvre de grappes d'innovations à la fois plus productives (et donc génératrices de croissance), plus sobres (et donc moins gourmandes en ressources) et plus écologiques (et donc moins polluantes). Le "développement durable" serait ainsi, comme le souligne justement F.-D. Vivien, une sorte de sixième étape de la croissance de Rostow, qui succéderait à celle de la "consommation de masse" » (Mayeur, 2005, p. 80).

Dans une perspective prudente, il est clair que l'humanité doit surtout miser sur les ressources renouvelables, mais elle peut avoir recours aux ressources épuisables dès lors qu'elle est capable de créer en même temps des biens capitaux de substitution :

«[On] peut formuler des principes entendus [...] comme des règles minimales de prudence :

(i) Les taux d'exploitation des ressources naturelles renouvelables doivent être égaux à leur taux de régénération.

(ii) Le taux d'émission des déchets doivent être égaux aux capacités d'assimilation et de recyclage des milieux dans lesquels ils sont rejetés.

(iii) L'exploitation des ressources naturelles non renouvelables doit se faire à un rythme égal à celui de la substitution des ressources renouvelables » (Daly, 1990, cité par Boisvert et Vivien, 2006, p. 36).

Pour les économistes d'inspiration néo-classique, la contradiction entre les deux termes «développement» et «durable» que soulignait Gilbert Rist n'existe pas. On peut concilier objectifs environnementaux (sauver la terre), objectifs sociaux (plus de justice) et objectifs économiques (poursuivre la croissance dans les pays du Nord, et la stimuler dans ceux du Sud). 


\subsection{Gestion des biens environnementaux et recours au marché}

Nombreux sont les économistes néo-libéraux qui pensent donc que la croissance peut se poursuivre de manière durable. Encore faut-il, pour y parvenir, que les rentes liées à l'exploitation des ressources naturelles soient effectivement affectées à la constitution d'un capital reproductible. Pour les ressources naturelles, comme le pétrole, la raréfaction de la ressource conduira à la hausse des prix : cela incitera les grandes compagnies à pousser la prospection dans des zones encore négligées, et à domestiquer d'autres formes d'énergie :

«La règle de Hotelling enseigne que le prix de vente des ressources non renouvelables ne doit pas être fonction des coûts d'extraction de ces ressources, mais de leurs stocks encore exploitables, de sorte que ce prix reflète la perte d'utilité des générations à venir qui ne pourront pas compter sur une matière première disparue. Ainsi, plus une ressource se fait rare, plus les consommateurs devront la payer cher quel que soit son coût de production » (Mayeur, 2005, p 75).

Comment prendre en compte, en revanche, ce que l'économie tire de l'exploitation de biens naturels libres, comme l'air ou l'eau ? C'est là un problème essentiel : tant que les industriels et les ménages n'en paient pas la consommation, ils ne les prennent pas en compte dans leurs calculs. Deux solutions sont alors envisageables : (i) la taxation, qui permet de prélever des sommes sur ceux qui polluent, et de les reverser à un fond destiné à améliorer la qualité des eaux et de l'air ; (ii) le recours au marché.

C'est cette seconde solution qui a la faveur de beaucoup d'économistes libéraux. Depuis l'article de Garett Hardin sur le paradoxe des biens communs (Hardin, 1968), on sait que la privatisation constitue un moyen efficace pour responsabiliser les acteurs économiques, car ils ne peuvent plus faire peser sur d'autres les coûts qu'engendrent leurs propres décisions.

«La privatisation présente cet avantage d'inciter les propriétaires de l'environnement à gérer de manière responsable la ressource rare [...].

Ici, c'est donc à travers le marché, en parlant au porte-monnaie et non à la bonne conscience des individus, qu'on les conduit à être écologiquement responsables [...]. L'idée de possible (et efficace) privatisation de la nature n'est pas nouvelle. Dès 1960, Ronald Coase montrera que, même pour des biens a priori publics [...] tels que l'eau et l'air, l'Etat peut délivrer des droits de propriété privée [...]. La seule manière fructueuse et significative de traiter des externalités est de les reconnaître stricto sensu comme des problèmes de droit de propriété.

L'externalité négative ne serait plus la manifestation d'un défaut du marché, mais d'un défaut de marché » (Lenglet, 2005, p. 230).

Comment privatiser un bien libre ? La réponse est fournie en 1968 par l'économiste canadien John Dales : il ne propose pas de vendre l'air, mais de créer un marché où vendre et acheter des droits d'émission pour les gaz à effet polluant (Dales, 1968).

«Un système de permis d'émission négociables est un instrument administratif de régulation dans lequel on introduit de la flexibilité pour permettre aux acteurs privés de s'adapter aux moindres coûts aux objectifs environnementaux fixés par la puissance publique, celle-ci étant dans l'incapacité d'affecter à chacun des 
contraintes justement réparties, c'est-à-dire de garantir une situation où aucun des acteurs n'est indûment pénalisé » (Aubertin, Hourcade et Vivien, 2006, p. 64).

Les achats et cessions déterminent des prix par rapport auxquels les industriels peuvent faire des choix rationnels : maintenir une fabrication polluante si les droits à acquérir ne pèsent pas trop sur leur prix de revient, ou se désengager et développer de nouvelles productions.

« Un tel mécanisme n'a pas grand-chose à voir avec un "marché" habituel ; $[.$. il s'agit plutôt d'une procédure de planification [...] à laquelle se trouve associé un système d'échange de quotas attribués pour une période donnée » (Aubertin, Hourcade et Vivien, 2006, p. 64).

Le marché peut-il aussi assouplir le jeu des contraintes que la volonté de préserver la biodiversité introduit aussi dans la vie de l'économie ? Sur un point, cela est réalisable : la biodiversité est un avantage qui bénéficie aux firmes pharmaceutiques qui mobilisent les connaissances botaniques de populations primitives pour détecter des molécules performantes dans la lutte contre telle ou telle maladie. Ces connaissances ont jusqu'ici étaient considérées comme des biens libres. Ne peut-on les traiter comme toutes les autres connaissances, c'est-à-dire leur attacher des droits de propriété intellectuelle ?

\subsection{L'arrière-plan de la Conférence de Rio de Janeiro}

Le contexte que nous venons d'évoquer fait comprendre les options retenues par la Conférence de Rio : on y retrouve la volonté des pays du Sud de sortir de la pauvreté, la mauvaise conscience des populations du Nord, qui reconnaissent leur responsabilité dans la dégradation de l'environnement planétaire, la nécessité pour les entreprises que la globalisation soumet à une concurrence intense de jouer sur toutes les formes de progrès ; les transformations de la réflexion écologique, sociale et économique aident à formuler les solutions.

Dans les années 1980, la mode est à la dérégulation : pour les hommes d'Etat, c'est une façon de montrer qu'ils ont compris le sens des évolutions en cours sur la scène mondiale. La globalisation est en marche. Comment planifier dans le détail le fonctionnement de systèmes complexes, et qui sont à cheval sur une pluralité d'Etats ? Les mesures de coordination internationale qu'implique la préservation de la nature doivent mettre en œuvre des moyens plus flexibles que ceux jusqu'alors mobilisés par la planification économique au sein des Etats.

Dans le mouvement des idées concernant la survie de l'environnement, les associations ont joué un rôle central depuis le début des années 1980. On commence à les qualifier d'ONG. Elles tiennent une place essentielle dans la préparation de la Conférence (Soyez, 2001).

\section{La Conférence de Rio de Janeiro}

La Conférence que l'ONU tient à Rio du 3 au 14 juin 1992, et qui constitue le premier Sommet de la Terre, est une énorme machine (on parle de 40000 participants) montée par Maurice Strong. Ce qu'elle apporte, ce sont d'abord deux grands principes : 
«Principe 1 : Les êtres humains sont au centre des préoccupations relatives au développement durable. Ils ont droit à une vie saine et productive en harmonie avec la nature.

Principe 4 : Pour parvenir à un développement durable, la protection de l'environnement doit faire partie intégrante du processus de développement et ne peut être considérée isolément » (Déclaration de Rio de Janeiro, citée par Voisenet, 2005, p. 17).

La Conférence propose ensuite la mise en œuvre d'un Agenda pour le $\mathrm{XXI}^{\mathrm{e}}$ siècle, l'Agenda 21. Il se décline sous plusieurs formes, car il est destiné à orienter à la fois les politiques des gouvernements et celles des autorités locales.

La Conférence se solde surtout par la signature de conventions. Dans le langage de l'ONU, « une convention est un traité international qui lance un processus de négociation sur sa traduction pratique. Ce processus a une vie propre, soumis aux aléas du cycle de la vie politique interne à chaque pays, des mouvements de l'opinion publique, des jeux médiatiques et des compromis diplomatiques tactiques » (Aubertin, Hourcade et Vivien, 2006, p. 58). Rio de Janeiro met ainsi en branle une dynamique qui fait du développement durable une des préoccupations majeures de tous les gouvernements. C'est à Rio, et durant les négociations qui jalonnent la mise en œuvre des conventions, que des méthodes sont définies pour mettre en œuvre les principes. L'action est confortée par la tenue d'un Deuxième Sommet de la planète Terre, à New York, en 1997.

Quatre conventions sont négociées durant la Conférence de Rio. Deux sont signées immédiatement, celles qui concernent les évolutions les plus menaçantes pour l'environnement global : la Convention sur le changement climatique, qui doit permettre de réduire l'émission de gaz à effet de serre, et la Convention sur la diversité biologique, qui doit mettre en œuvre tout ce qu'il est possible de faire pour maintenir la biodiversité. Une troisième convention, celle sur la désertification, est ratifiée deux ans plus tard. La quatrième négociation, qui portait sur la protection des forêts, échoue.

\subsection{La Convention sur le changement climatique}

Comment mettre en œuvre les principes retenus à Rio par la Convention sur le changement climatique ? On dispose en ce domaine de données relativement précises sur les émissions de gaz à effet de serre dont sont responsables les divers pays du monde : celles-ci reflètent la consommation de combustibles fossiles par les entreprises et par les ménages, certaines formes d'exploitation rurale (l'élevage bovin par exemple, les vaches émettant des quantités importantes de méthane), et la déforestation par le feu.

Pour réduire l'impact du développement sur l'environnement, il convient donc d'économiser l'énergie, surtout lorsqu'elle est d'origine fossile, de ralentir ou de stopper la déforestation, et d'encourager la plantation de végétaux capables de stocker des masses importantes de carbone. A partir des bases chiffrées dont on dispose, on peut négocier des plans de réduction des consommations, qui permettront de stopper, puis de faire régresser la contribution de chaque pays au danger global. Par le protocole de Kyoto, signé en décembre 1997, les pays de l'OCDE, les pays de l'Europe de l'Est, la Russie et l'Ukraine s'engagent à réduire pour la période 2008-2012 leurs émissions gaz à effet de serre de 5,2\% par rapport à ce qu'elles étaient en 1990. La portée du protocole est limitée par le refus des Etats-Unis de souscrire à ces engagements, mais le processus est lancé. La mise en place de marchés de 
droits d'émission de gaz polluants introduit une certaine souplesse dans le fonctionnement du nouveau système réglementaire. Elle alimente des circuits de financement : les sommes versées par les pollueurs dans les pays qui manquent de droits peuvent être achetés dans des contrées qui n'utilisent pas les leurs, et contribuer ainsi à leur développement.

\subsection{La Convention sur la diversité biologique}

Les problèmes que pose la mise en œuvre de la seconde grande convention signée à Rio de Janeiro sont très différents : il n'existe pas de mesure de la biodiversité. Le nombre des espèces en constitue sans doute la meilleure approximation, mais son établissement demande de longues études; l'inventaire qu'il implique est loin d'être achevé dans une bonne partie du monde - dans les régions tropicales en particulier. Faut-il passer de la diversité des êtres à la diversité des gènes ? C'est le pari qui est fait :

« [Le but de la Convention] est la conservation de la diversité biologique, l'utilisation durable de ses éléments et le partage juste et équitable des avantages découlant de l'exploitation des ressources génétiques, notamment grâce à un accès satisfaisant aux ressources génétiques et à un transfert approprié des techniques pertinentes, compte tenu de tous les droits sur ces ressources et sur ces techniques, et grâce à un financement adéquat » (art. 1).

La formulation retenue porte la marque du passage de la perspective naturaliste à la perspective biologique. Elle témoigne aussi de l'influence des techniciens du développement agricole - très sensibles aux problèmes de la sélection des espèces - et des industriels de la pharmacie - d'où l'inclusion dans les dispositifs de la Convention d'un mécanisme de marché :

«Le "marché" attendu par les instances de régulation dans le cadre de la CDB

(Convention sur la Diversité Biologique) reconnaît des droits de propriété intel-

lectuelle préalables à la commercialisation des ressources biologiques » (Aubertin,

Hourcade, Vivien, 2006, p. 67).

Pour établir une politique en faveur de la biodiversité, il n'est pas possible de partir d'un bilan mondial pour répartir ensuite des droits entre les différents États : ce que l'on veut préserver n'est pas encore mesuré, et n'est peut-être pas objectivement mesurable. Force est de reconnaître que la responsabilité qui revient aux Etats, aux autorités locales, aux entreprises et aux ménages est beaucoup plus directe qu'en matière de pollution atmosphérique.

Les formulations choisies ont été pensées pour les nouvelles biotechnologies : on garantit aux entreprises qui les développent le droit d'utiliser les molécules que d'autres ont découvert, moyennant le paiement de droits raisonnables. Les firmes des pays industrialisés financeront de la sorte la croissance des régions où elles vont chercher les substances dont elles industrialisent la production.

L'accent mis sur les ressources génétiques explique aussi la place que les OGM (Organismes Génétiquement Modifiés) tiennent dans les négociations que mène la Convention.

Pour l'essentiel cependant, la mise en œuvre de celle-ci implique une stratégie ascendante : il faut partir des observations de terrain, des dangers qui pèsent ici ou là sur tel 
milieu ou sur tel autre, pour cerner les problèmes et envisager des solutions. Que celles-ci impliquent des mesures de niveau national ou international, c'est évident : il faut réduire l'usage des pesticides, contrôler celui des engrais, élaborer des systèmes juridiques de protection plus adaptés à tel ou tel environnement. Ces mesures sont là pour répondre à des menaces précises, localisées : c'est de leur somme que résultera l'effet souhaité, à savoir le maintien de la biodiversité globale.

\subsection{Perspective sur le processus de Rio}

La signification du Sommet de la Terre de Rio vient du processus qu'il met en marche : les problèmes d'environnement sont désormais pris en compte au même titre que les problèmes sociaux et les problèmes économiques dans la définition des politiques menées au niveau local, au niveau national et au niveau global. La tradition voulait que les questions de milieu soient surtout considérées comme des problèmes circonscrits à certains lieux ou à certaines zones. Ils ne peuvent plus être réglés en opérant de la sorte. Ils ne peuvent plus l'être en ignorant la pauvreté : comment protéger des milieux indispensables à la survie de gens que la famine menace?

Telle qu'il était conçu par l'écologie profonde qui se développe dans les années 1970 et soutient que les animaux, les végétaux et la nature inanimée doivent être dotés de droits que les hommes doivent respecter, le tournant environnemental risquait d'être uniquement conçu en termes de restrictions et de limitations. Ce qu'introduit le processus de Rio est différent :

«Le propre du développement durable, ce n'est pas la conservation, mais l'évolution, la mutation des équilibres, une adaptation aux nouvelles conditions [...]. Le développement durable doit viser à améliorer le développement des générations à venir » (Skiada, cité par Cobast, 2005, p. 37).

Sylvie Brunel le souligne autrement, lorsqu'elle dit :

"C'est une trilogie où l'économie (produire plus mais au service du plus grand nombre) s'allie au social (répartir mieux, lutter contre la pauvreté) dans le respect de l'environnement (préserver l'avenir et les conditions de vie des générations futures) qui fonde les trois composantes essentielles du développement durable » (Brunel, 2005, p. 122).

L'ONU a d'autre part introduit un mécanisme nouveau et fondamental : les conventions conduisent les Etats à coopérer pour limiter le rejet des gaz à effets de serre et pour sauvegarder les espèces.

«Projet politique, le développement durable est contestataire. Il met en cause l'ordre existant. Pourquoi faudrait-il insister sur les responsabilités à l'égard des "générations futures" si le cours actuel des choses devait mener à coup sûr vers un avenir meilleur? » (Smouts, 2005, p 7-8).

Les réflexions relatives à la nature, à la société et à la croissance qui sont menées à partir des années 1970 sont mises en œuvre par la Conférence de Rio de Janeiro qui en tire les conséquences sur le plan politique et au niveau international. Beaucoup d'États avaient 
développé, depuis les années 1960 surtout, des politiques environnementales ambitieuses. Ils ne les avaient pas toujours liées à la question de l'essor économique et social de leurs populations. Ils ne s'étaient pas souciés de ce qui se passait hors de leurs frontières - ce qui est pourtant essentiel dès lors que l'économie se mondialise et que des menaces globales pèsent sur l'environnement. On mesure à cela l'apport de la Conférence de Rio de Janeiro.

\section{D'une réflexion venue du haut à une explosion tous azimuts}

Avec le Sommet de la Terre, l'idée de développement durable échappe à ceux qui l'ont lancée ; elle circule dans la société ; elle est reprise par Monsieur Tout le Monde, par les chefs d'entreprise, par les hommes politiques. Elle sert de fond de commerce à de multiples associations et ONG. C'est le visage qu'elle prend ainsi qu'il convient de cerner.

\subsection{Une élaboration qui s'est faite au sommet}

Les politiques de croissance telles qu'elles sont pratiquées dans les années 1960 créent un malaise dans les pays riches : « les sociétés industrielles développées nourrissent [...] un fort sentiment de culpabilité car les atteintes à l'environnement qu'elles génèrent ont pris une dimension mondiale » (Lenglet, 2005, p. 265). Les tensions s'accroissent entre les pays en voie de développement (on parle trente plus tard des pays du Sud) et les pays industrialisés (aujourd'hui qualifiés de pays du Nord) : la détérioration des termes de l'échange, à peu près constante durant les années 1950 et 1960, donne aux premiers le sentiment que la fin du colonialisme n'a pas arrêté l'exploitation à laquelle ils attribuent leur retard. Ils ont donc tendance à rejeter la responsabilité de leur stagnation sur l'Europe, les États-Unis et dans une certaine mesure le Japon : ils aimeraient bénéficier de transferts plus substantiels.

Le sentiment que l'environnement se détériore est largement partagé : il est d'autant plus sensible que la pollution atmosphérique, de plus en plus sensible dans les villes un peu partout dans le monde, est perçue par tous. Les allergies se multiplient. Les enfants ont davantage de bronchites et deviennent plus souvent asthmatiques.

Le tournant de la réflexion sur la croissance qui intervient au début des années 1970 s'ancre donc dans des insatisfactions largement partagées par les populations aussi bien au Nord qu'au Sud - même si les motifs ne sont pas toujours les mêmes. La réflexion qui en découle est menée par des intellectuels, dont beaucoup se recrutent en Amérique du Nord, en Grande-Bretagne, en Allemagne ou dans les pays scandinaves. Les pays latins sont moins directement associés au mouvement à la fois pour des raisons linguistiques - la langue dans laquelle s'expriment inquiétudes et propositions est l'anglais - et idéologiques - le protestantisme constituant sans doute un meilleur terreau pour les nouveaux courants de pensée que le catholicisme.

Comment légitimer alors l'idée de développement durable ? En montrant qu'elle a des racines populaires. C'est ce qui explique la faveur dont jouissent alors les associations qui prennent désormais de plus en plus souvent la forme d'organisations non gouvernementales : en étendant leur champ d'activité, elles gagnent un nouveau statut. Elles n'unissent plus simplement des naturalistes et des hommes de bonne volonté un peu partout dans le monde : elles ont une assise internationale qui leur permet de parler 
au nom des foules anonymes qui subissent les malfaçons de la croissance et de la globalisation sans avoir voix au chapitre. Elles s'arrogent aussi le droit de parler au nom des araignées, des vers de terre, des moineaux ou des éperviers que l'ouverture de nouvelles routes et l'urbanisation dérangent ou font disparaître. Dietrich Soyez le dit fort bien :

«Les processus de globalisation sont souvent compris comme des secousses venant du haut et qui transforment profondément les structures, les processus et les acteurs présents au niveau local. Au cours des dernières années, il est devenu clair que les processus qui remontent de la base au sommet sont en train de prendre de l'importance dans la mesure où leur expression arrive désormais à cheminer d'un échelon à l'autre, et jusqu'au sommet. Les organisations non-gouvernementales (désignées ici sous le nom de lobbies) et les réseaux transnationaux de défense des causes [Transnational Advocacy Networks, en abrégé TAN] sont des signes clairs de cette transition. Le nombre et l'influence de ces lobbies a cru dramatiquement au cours de ces récentes années, et comme leur activité se joue de plus en plus des frontières, ils constituent aujourd'hui les acteurs les plus influents dans le processus de passage du local au global » (Soyez, 2001).

Soyez rejoint sur ce point le diagnostic de Tellenne :

«Les ONG [Organisation Non Gouvernementales] sont [...] des acteurs majeurs du développement durable : elles incarnent le "tiers secteur', c'est-à-dire qu'elles ne poursuivent ni profit ni objectifs gouvernementaux, d'où leur légitimité apparente » (Tellenne, 2005, p 269).

On comprend pourquoi la présence massive des ONG n'a soulevé aucune protestation lors de la préparation su Premier Sommet de la Terre à Rio : elles assuraient, par leur présence, la légitimité du processus en cours.

L'ambiguïté d'une telle situation est évidente : c'est parce que les ONG passent pour représenter la multitude de ceux qui n'ont pas la parole que la construction de l'idée de développement durable échappe aux critiques et ne paraît pas résulter d'une démarche purement technocratique. En fait, les ONG ne représentent qu'elles-mêmes, c'est-à-dire des groupes faits de chercheurs convaincus d'agir pour la sauvegarde de l'environnement et le bonheur de l'humanité, et de militants acquis à leurs idées.

Au total, l'idée de développement global est née dans des sphères intellectuelles assez limitées et s'est imposée comme légitime à la Conférence de Rio car elle passait pour émaner des couches profondes de la société. Les politiques qui en découlent suivent dans une large mesure la même voie.

\subsection{Une nouvelle dimension des procédures descendantes : l'irréversibilité des évolutions en cours}

L'espoir de ceux qui lancent l'idée de développement durable, dans les années 1980, c'est d'imaginer des politiques susceptibles de restaurer l'ordre naturel et d'arrêter des processus irréversibles, celui du changement climatique en particulier. Au fur et à mesure que les connaissances se précisent, la réalisation d'un tel objectif paraît de plus en plus 
utopique : une grande partie de l'humanité est encore pauvre; il est impossible de lui interdire d'accéder à des niveaux de vie meilleurs, ce qui accroîtra considérablement les consommations d'énergie, de denrées alimentaires et de matières premières. L'effet des politiques de préservation n'est d'autre part pas immédiat : si l'on arrivait, par on ne sait quel miracle, à arrêter l'émission des gaz à effet de serre, leur accumulation dans l'atmosphère demeurerait à un niveau si élevé que durant au moins trente ans, la dégradation des climats continuerait sur sa lancée.

Le Conseil International sur le Changement Climatique joue un rôle essentiel en ce domaine : c'est l'organisme qui conseille les Nations Unies. Dominé par des spécialistes de la dynamique de l'atmosphère, il a la responsabilité d'organiser la collecte des données relatives à l'évolution du climat; il les incorporent dans des modèles qui permettent d'élaborer des projections valables pour le futur. Celles-ci varient largement - la hausse prévue des températures pour le $\mathrm{XXI}^{\mathrm{e}}$ siècle fluctue, selon les cas, entre $1^{\circ} \mathrm{C}$ et $5,4^{\circ} \mathrm{C}$. Quel est le pronostic le plus valable ? La prudence s'impose, mais lorsque les avis convergent, le Conseil International en prend acte et formule des avis qui guident l'action internationale : celui qui s'est imposé au cours des dernières années, c'est que le changement climatique est une réalité, et qu'il a un rythme tel (même s'il n'est pas possible de le préciser) que le retour à l'état initial est impossible. La finalité des politiques change donc : il ne s'agit plus de dire comment fonctionnent les écosystèmes pour les préserver. Ce qu'il importe maintenant de savoir, c'est comment les milieux existants pourront s'adapter aux mutations prévisibles, de manière à les prévoir et à en éviter les conséquences les plus dramatiques. Le Conseil International du Changement Climatique demande donc à chaque pays de procéder à des études scientifiques sur les effets que le changement global aura dans ses limites, et sur les mesures à prendre pour faciliter les évolutions que l'on peut y prévoir.

C'est l'aspect dramatique des prévisions climatiques qui justifie la place donnée à la procédure descendante. C'est lui qui explique que l'on préfère à l'analyse précise des conditions présentes les scénarios qui permettent de dessiner le visage du futur.

\subsection{Multiplication des acteurs et dérive des thèmes}

Une fois lancé, le développement durable implique tous les acteurs des jeux sociaux, économiques et politiques qui se déroulent à la surface de la terre : (i) il demande aux individus d'être des citoyens du monde conscients des dangers qui pèsent sur l'ensemble de l'humanité, des consommateurs qui contrôlent leurs achats et les déchets qui en résultent, et des travailleurs prêts à mettre en œuvre des techniques nouvelles, et peut-être moins économes de temps et d'efforts, pour le bien de tous ; (ii) il oblige les entreprises à revoir leurs objectifs et leurs pratiques si elles veulent sauvegarder leur bonne image dans une société qui tolère de moins en moins les pollutions ; (iii) il repose sur une implication de tous les échelons des systèmes politiques et administratifs, du niveau global au niveau local, et sur l'invention de procédures qui permettent une coopération efficace entre ces divers échelons.

Le succès des actions de développement durable repose sur l'adoption d'attitudes nouvelles : il implique donc une transformation des liens symboliques qui existent dans les sociétés. Les intellectuels ont dans ce domaine une responsabilité directe. Leur travail serait vain s'il n'était relayé par les médias qui le font connaître, par l'enseignement qui 
le transmet aux classes montantes, et par des associations qui permettent à la réflexion de s'épanouir à tous les échelons et de rebondir d'un niveau à l'autre.

Les différents acteurs du jeu social apprennent à intégrer les horizons spatiaux et temporels qu'implique la perspective du développement durable. « Du point de vue de l'entreprise », écrit Proglio (2005, p 259), « c'est le croisement d'une gestion prévisionnelle de tous les risques et d'une réflexion prospective sur les conséquences de l'action présente et les conditions d'exercice de l'activité de demain. En un mot, c'est la responsabilité ».

Le problème est pris particulièrement au sérieux par les industries lourdes, production d'énergie, sidérurgie, fabrication du ciment, pétroléochimie : elles émettent beaucoup de gaz à effet de serre et mettent sur le marché des denrées dont les effets sur l'environnement peuvent être nocifs s'ils ne sont pas biodégradables, s'ils contiennent des métaux lourds, s'ils polluent les eaux de surface ou profondes, comme les engrais, ou s'ils menacent la totalité de certaines chaînes trophiques, comme beaucoup de pesticides. A une phase initiale de rejet du développement durable a succédé, pour les industries de ces secteurs, une attitude plus ouverte et le lancement d'actions volontaires destinées à améliorer leur image (Ekman, 1998 ; Lefèvre, 2006). Des guides sont publiés pour les aider dans cette voie (ICC, 1991 WBCSC, 2001). Beaucoup d'entreprises se sentent écologiquement responsables :

«La responsabilité sociétale de l'entreprise (RSE) apparaît aujourd'hui comme la traduction microéconomique la plus visible du développement durable. Elle pourrait, dans les années qui viennent, se diffuser à la fois en aval, dans une direction microsociologique, vers ce que l'on pourrait appeler une RSI (responsabilité sociétale des individus) et en amont, dans une direction macrosociologique, vers une responsabilité sociétale des États. L'actualité plus ou moins récente suggère cette diffusion : le sang contaminé, la canicule de l'été 2003, la vache folle, l'amiante, la Charte de l'Environnement..., les États semblent de plus en plus mis en cause dans ces affaires » (Sainteny, in Smouts, 2005, p. 202).

La place tenue par la presse est essentielle :

«La défense de l'environnement est apparue, dès les années 1970, comme une cause particulièrement portée par l'univers médiatique. Elle présente en effet, à la manière de l'humanitaire par exemple, tous les traits de la cause sans adversaires, appuyée sur un fort investissement moral, la mise en avant du désintéressement et le recours à des collectifs (l'état de la planète, les “générations futures'..) qui intéressent les journalistes » (Riutort, 2005, p. 99).

La presse permet au mouvement pour l'environnement de revêtir un caractère global (McCormick, 1995). Les organisations non gouvernementales (ONG) qui sont à sa tête sont à la fois très critiques et prêtes à collaborer avec les entreprises et les gouvernements (Chartier et Ollivraut, 2006).

Les médias se font volontiers les interprètes des chercheurs qui s'investissent alors dans l'environnement. Ils jouent un rôle essentiel dans la diffusion de leurs idées. Ils y sont d'autant plus sensibles que les prises de position de beaucoup de savants ont une forte connotation éthique. Les journalistes la renforcent : ils participent ainsi, de conserve avec les spécialistes du milieu, à la naissance de nouvelles idéologies, celles que l'on désigne souvent sous le nom d'écologisme. 
«Le développement des moyens de communication et le progrès de la recherche scientifique concourent à restaurer une situation qui évoque celle qui prévalait dans les sociétés archaïques. Dans les deux cas, les gens ordinaires désirent avoir des informations sur une réalité qui leur paraît riche de signification, mais à laquelle ils n'ont pas directement accès [...]. Dans les sociétés contemporaines, la plupart des gens sont dans l'incapacité d'évaluer eux-même la portée des résultats scientifiques. Ils n'ont accès qu'aux interprétations que les journalistes en proposent. [...] Ces intermédiaires possèdent ainsi un large pouvoir de remodeler l'information » (Claval, 2001, p. 150).

L'idée de développement durable rencontre d'autant plus d'échos dans la population, chez les chefs d'entreprise ou chez les responsables politiques qu'elle s'est vue ainsi lestée d'une dimension morale (Claval, 2000). Pour parodier un mot célèbre du général de Gaulle, ce n'est plus un concept, c'est « une ardente obligation morale».

\section{Stratégies descendantes et stratégies ascendantes}

\subsection{Une mise en æuvre qui part largement du niveau global}

Les politiques mises en œuvre pour assurer le développement durable diffèrent par les échelles où l'on situe les tensions qui déstabilisent les relations hommes/nature : le rapport Brundtland met au premier plan l'émergence de déséquilibres globaux (émission de gaz à effets de serre, et accumulation de gaz affaiblissant la couche d'ozone). Mais ces déséquilibres globaux résultent d'actions menées partout à la surface de la terre : celles qui conduisent à l'émission des gaz à effet de serre, l'oxyde de carbone et le méthane essentiellement, et celles qui concernent les gaz de composition fluoro-chlorée.

La stratégie conçue par le rapport Brundtland et mise en œuvre depuis part d'observations menées au niveau global et en déduit les actions qu'il convient de mener au niveau national, au niveau régional et au niveau local pour éviter de nuire à l'environnement. Pour réduire l'émission des gaz à effet de serre, il convient de maintenir le couvert forestier là où il existe, et de le reconstituer là où il a disparu ; il faut limiter les consommations d'énergie fossile et développer l'usage d'énergies renouvelables et, peut-être, de l'énergie nucléaire. Pour réduire les menaces sur la couche d'ozone, il faut renoncer à l'emploi de composés fluoro-chlorés dans les équipements industriels ou domestiques.

Les politiques élaborées sous l'égide des Nations Unies partent donc des déséquilibres globaux pour obtenir, par des actions localisées, une réduction des tensions environnementales. Dans le cas de l'Europe par exemple, des directives ont été élaborées par Bruxelles en vue de la réalisation de ces objectifs. La ratification du protocole de Kyoto signifie que chaque pays doit avoir, en 2012, réduit de $8 \%$ le niveau d'émission qui était le sien en 1990. Dans cette optique, chaque aire doit réduire sa contributions à l'émission de gaz à effets de serre ou de composés fluorés pour parvenir à la réduction des tensions globales. La mise en place d'un système d'échanges des droits d'émission de gaz à effet de serre doit faciliter la réalisation de l'objectif européen global.

Pour mener à bien ces actions, il convient de disposer d'indicateurs qui précisent la responsabilité de chaque lieu dans le déséquilibre global. Un exemple : en ce domaine, la Région Ile-de-France met en œuvre un indicateur de «l'empreinte écologique de ses habitants ». 


\subsection{Stratégies sectorielles, stratégies localisées, stratégies ascendantes}

1- A cette stratégie, qui part du constat de déséquilibres globaux et met en œuvre des actions régionalisées pour les éliminer, s'en ajoutent d'autres, qui prennent en compte l'existence de déséquilibres sectoriels : ceux-ci se traduisent par des pénuries particulières (l'eau par exemple), ou par l'utilisation trop forte de produits polluants (les pesticides) ou qui peuvent le devenir (les engrais).

Des travaux importants ont trait liés aux problèmes de l'approvisionnement en eau, et aux actions à mener pour réduire les pénuries en ce domaine : contrôle des utilisations de l'eau de qualité et recyclage systématique des eaux usées; recherche de ressources nouvelles; lutte contre les pénuries par les transferts directs d'eau (barrages, canaux ou conduites) ou les transferts indirects (en réorientant les activités économiques de façon à exporter des produits ou des services qui incorporent peu d'eau, ou demandent peu d'eau pour leur fabrication ou leur mise en œuvre, contre des produits ou des services qui en consomment beaucoup).

2- Certains environnements sont particulièrement fragiles, ce qui implique des actions spécifiques : les eaux du lac Baïkal sont encore d'une extrême pureté, mais il suffirait de peu pour y provoquer des transformations irréversibles ; l'assèchement de la mer d'Aral impose une révision des politiques de l'eau sur l'ensemble des bassins de l'Amour Daria et du Syr Daria. Les mers quasi fermées, Baltique et Méditerranée, mer Noire, mer Rouge, nécessitent aussi des actions particulières.

3- Comme on vient de le rappeler, les politiques de développement durable sont généralement construites à partir du constat de déséquilibres écologiques. Une approche alternative part de l'analyse des milieux et de leur fonctionnement. Les environnements humains se transforment rapidement dans le monde actuel. La suburbanisation généralisée multiplie les jardins, les espaces verts et les aires aménagées pour les pratiques sportives. Comment évaluer la pression écologique que les hommes exercent sur de tels milieux ? Comment la comparer à celle qu'ils exerçaient lorsqu'ils vivaient dans des milieux plus traditionnels et plus denses ? Pour rendre possible le développement durable, ne convient-il pas de sélectionner les formes d'aménagement de l'espace qui gaspillent le moins d'énergie et de ressources rares ? L'analyse des flux de matière et d'énergie dans les différents milieux constitue ainsi un moyen fondamental de connaissance des déséquilibres environnementaux (Buttimer et Stol, 2001).

L'opposition entre ville, campagne et nature sauvage rendait bien compte, il y a encore trois-quarts de siècle, de l'organisation des espaces géographiques. Les types dominants ont changé : espaces densément urbanisés ; espaces urbanisés lâches ou suburbanisés ; rural ou pastoral profond ; ensembles « naturels » reconstitués et gérés par les autorités écologiques. Ces transformations résultent de la possibilité de mettre partout en œuvre des formes concentrées d'énergie, de disposer de moyens de transport qui élargissent les aires de migrations quotidiennes ou de déplacements périodiques, et d'avoir accès aux réseaux d'acheminement et de traitement des informations. Les milieux qui résultent de ces évolutions ne seraient pas viables sans le nouvel environnement technique. Les formes qu'ils revêtent résultent des idéaux et des modèles de vie qu'ont adoptés les hommes politiques et les promoteurs qui les dessinent, et les gens qui les habitent. 
Dans quelle mesure la genèse de ces nouveaux environnements ne traduit-elle pas aussi les incohérences des systèmes de prix en œuvre dans les sociétés actuelles - la facturation à un prix trop faible de l'énergie, par exemple ? Ne convient-il pas, pour assurer un développement durable, de mettre en place des systèmes d'indicateurs économiques, d'incitations et de prix dont les effets soient plus « écologiques »?

\subsection{Développement durable et opinion publique}

Les actions visant à assurer un développement durable sont nées d'observations scientifiques concernant l'évolution du climat à l'échelle globale. Elles sont traduites en termes de programmes d'aménagement par des experts travaillant dans le cadre des Nations Unies, de l'Union européenne ou des Etats.

Ces actions doivent être mises en œuvre par les Etats et par les collectivités territoriales. Elles le sont d'autant mieux que les élus et les gouvernants comprennent les objectifs nouveaux qu'on leur propose, et y adhèrent. A l'échelon des communautés locales, par exemple, les élus se sont familiarisés, il y a une génération, avec les plans d'utilisation des sols. Il leur faut maintenant veiller à limiter l'empreinte écologique des habitants dans les espaces qu'ils gèrent : le leur faire admettre prend du temps et implique des actions pédagogiques, dont il convient de suivre et de mesurer l'efficacité.

L'idée de développement durable est aussi interprétée par le citoyen ordinaire : c'est parce que celui-ci a fait sien l'idéal d'une humanité réconciliée avec les milieux qui la font vivre que les actions politiques à visées écologiques ont des chances d'aboutir. Mais l'idée de développement durable telle qu'elle est comprise par le citoyen ordinaire ne coïncide pas avec celle qu'on en a en haut lieu. Les échelles prises en considération ne sont pas les mêmes : le développement durable pour lequel on est prêt à lutter est celui qui rend plus agréable l'environnement proche et y évite l'installation d'activités génératrices de nuisance. L'idée de développement durable fortifie souvent les égoïsmes locaux.

«L'acronyme Nimby, qui signifie littéralement « Not In My BackYard», traduit en français par «Pas de ça chez moi ! » est censé illustrer les paradoxes, voire les contradictions qui caractériseraient les défenseurs de l'environnement. Opposés par principe à la présence à proximité de leurs lieux de vie d'installations présentant des risques pour la santé publique (lignes à haute tension, centrales nucléaires....) ou certains désagréments (notamment le bruit et la modification des paysages pour les tracés de lignes TGV), ils s'en accommoderaient fort bien à partir du moment où les éventuels dommages seraient supportés par d'autres » (Ruitort, 2005, p. 94).

Les objectifs globaux ne sont généralement pas pris en compte par la base. On s'en rend compte en analysant la manière dont l'Agenda 21 local est mis en œuvre dans les villes françaises, Angers par exemple :

«La ville d'Angers [...] a été l'une des premières en France à mettre en place un Agenda 21 local. Ce programme d'action est conçu autour de quatre axes majeurs d'intervention :

- la solidarité à la fois locale - améliorer les conditions de vie des quartiers défavorisés à Angers - et internationale - contribuer à l'accès à l'éducation et à la santé des enfants de Bamako au Mali ; 
- la préservation des ressources et du cadre de vie, avec des mesures de lutte contre les nuisances sonores et pour la qualité environnementale des bâtiments publics [...] ; - la valorisation du territoire d'Angers, avec pour priorité de mettre en cohérence les dispositifs réglementaires [...] ;

- la mobilisation de tous les acteurs du territoire avec [...] l'association des citoyens aux économies d'énergie, à la réduction de l'usage des pesticides, au commerce équitable et aux enjeux planétaires du développement durable » (Sacquet, 2002, p. 55-56).

L'effort d'ouverture est certain, mais les objectifs de « qualité de la vie » pèsent autant que ceux de développement durable.

«Le rapport Brundtland fixait une définition souple du développement soutenable ou durable. On peut constater que, appliquée à la gestion et à la promotion des territoires, elle est facilement élargie et interprétée : ce serait tout ce qui peut permettre de fixer sur un territoire (souvent assez restreint) des ressources susceptibles d'alimenter le développement. Incontestablement, l'idée, partie de la défense un peu convenue de l'environnement global, évolue. Elle doit ses succès actuels plutôt à un certain égoïsme qu'à la claire conscience de la finitude des ressources terrestres » (Ferrandéry, 2005, p 199).

\subsection{Développement durable et idéologies}

L'idée de développement durable se nourrit d'idéologies, qui cherchent à culpabiliser les hommes parce qu'ils endommagent l'environnement. C'est que souligne William Rees, comme le rappelle Tellenne :

«Selon William Rees, "la durabilité exige de déplacer l'accent sur la gestion des ressources à la gestion de nous-mêmes". La mobilisation du public en faveur du développement durable revêt ainsi trois formes essentielles.

La première est la consommation "écocitoyenne" et "durable" à travers le commerce équitable [...].

La deuxième est la réalisation de gestes quotidiens respectueux de l'environnement. [...] Le tri sélectif des déchets devient un nouvel acte de civisme [...].

La troisième forme concerne les actions caritatives comme les dons ou le bénévolat international [...].

Le développement durable s'affirme ainsi comme un nouveau besoin social pour des populations angoissées par la finitude des ressources naturelles qui ont été au fondement de leur rapide enrichissement, par la mise en danger des grands équilibres planétaires, par les atteintes les plus manifestes à leur cadre de vie » (Tellenne, 2005, p. 275

Le tri des ordures est indispensable pour permettre leur recyclage. Mais les contrôles tatillons auxquels il donne lieu dans certains pays, l'Allemagne par exemple, ne sont pas seulement motivés par la recherche de l'efficacité : ils ont pour but d'imposer un contrôle de la communauté sur les comportements individuels et d'ostraciser ceux qui ne se plient pas de bon gré aux nouvelles disciplines collectives. Des recherches sont menées en Suède pour imposer l'usage de toilettes qui isolent les urines des fesces. Le but est de diminuer la consommation 
en eau et de tirer le meilleur parti écologique des excréments humains. Une telle action est-elle réellement imposée par l'état du milieu, en Scandinavie en particulier, où une pénurie d'eau n'est guère à craindre dans l'immédiat ? Non, mais il existe une autre motivation : l'utilisation de ces toilettes écologiques transforme un acte naturel en un acte civique, puisque l'individu doit penser aux responsabilités qu'il a vis-à-vis de la nature même dans ses moments d'isolement. Le but poursuivi est plus moral qu'écologique : responsabiliser les populations en leur rappelant que tous leurs actes pèsent sur l'équilibre du milieu.

\section{Les échelles appropriées au développement durable}

Anne Buttimer définit parfaitement les problèmes géographiques que pose la mise en œuvre des politiques de développement durable :

«Le défi qui consiste à concilier trois motivations sociales profondes se trouvent au cœur du développement durable : la croissance économique, la vitalité sociale et l'intégrité écologique. Les tensions entre ces objectifs varient selon les époques et les cultures : pour chacun de ces domaines d'expérience, il doit exister un horizon d'échelle optimal. Il y a des échelles minimales d'efficacité fonctionnelle essentielles pour la croissance économique, car les entreprises ont à négocier une niche viable pour leurs produits sur les marchés nationaux ou globaux [...]. Des seuils minimaux de taille, de revenu et d'interaction sont également décisifs pour la vitalité sociale aussi bien à l'échelle locale que nationale [...]. Bien que les limites de tolérance concernant l'intervention humaine dans les processus naturels et les conditions minimales de bio-reproduction soient encore l'objet de débats passionnés, les écologistes sont aujourd'hui de plus en plus convaincus que les éléments humains doivent être inclus dans les définitions de l'intégrité des paysages. Pour que les paysages et les genres de vie soient durables, une échelle appropriée d'action et d'interaction doit être identifiée : une échelle qui permette aux intérêts ascendants et aux demandes venues du haut d'être négociés » (Buttimer, 2001, p. 7-8).

La manière de concevoir les intérêts économiques et sociaux en jeu a subi une transformation profonde :

«Au cours de la période 1950-1990, [...] les forces primaires qui poussaient au changement provenaient de la socio-technosphère, c'est-à-dire des institutions et de la technologie. En d'autres termes, les forces qui penchaient pour l'ordre dépassaient de beaucoup celles qui concernaient les identités et l'organisation des genres de vie de tous les jours. La question se pose donc de savoir quelle échelle d'organisation politique convient pour négocier les intérêts potentiellement conflictuels des différents genres de vie. Les critères relatifs au choix d'une échelle appropriée [au développement durable] devrait donc inclure l'horizon spatial et administratif qui permettrait de négocier à la fois les intérêts mis en jeu par l'identité, par l'ordre et par les niches que génèrent les différents genres de vie » (Buttimer, 2001, p. 23).

Anne Buttimer précise plus loin sa pensée :

«Trois échelles distinctes d'horizons de relations ont été identifiées. [...] En premier lieu se trouvent les échelles politiquement définies des relations administratives, du 
local au global, à travers lesquelles les diverses fonctions sociales et politiques sont normalement remplies. En second lieu se placent les échelles industriellement définies de relations fonctionnelles, telles que celles au sein desquelles les systèmes manufacturiers, les champs urbains et les réseaux de service exercent leur influence [...]. En troisième lieu, l'expérience quotidienne, le sens de l'appartenance et l'identification à un lieu sont intimement liés à l'échelle des espaces bien connus que l'on traverse de la maison au voisinage, à la ville et à la région » (Buttimer, 2001, p. 25).

Torsten Hägerstrand montre par un exemple combien le choix des échelles où concilier les divers intérêts en jeu est important pour le développement durable :

«La différence entre le discours abstrait de niveau général et la réalité concrète locale constitue un sérieux problème. Dans un système de sectorisation fonctionnelle, le risque est grand que les différents secteurs arrivent à des conclusions contradictoires au niveau du paysage où il convient de loger les activités concomitantes qui y sont en concurrence. Les relations de pouvoir risquent alors de s'imposer, au détriment de solutions équilibrées. Il ne fait pas de doute, par exemple, que le secteur hautement technocratique des transports a fait éclater en morceaux les bourgs et les villes d'Europe, parce que les secteurs responsables des intérêts sociaux, esthétiques et environnementaux étaient relativement faibles à cette époque » (Hägerstrand, 2001, p. 45).

\subsection{L'adaptation de la recherche au discours sur le développement durable}

L'idée de développement durable ne marque pas le début des travaux sur les relations hommes/milieux. Elle propose une formulation nouvelle adaptée à l'évolution sociale (l'aspiration au développement et à la justice) et écologique (l'apparition de menaces sur l'environnement global). Elle reflète l'évolution des connaissances relatives aux écosystèmes terrestres.

Le nouveau cadre retenu pour penser les rapports des sociétés à la nature influe sur la conduite de la recherche. (i) Les chercheurs et les institutions de recherche doivent reformuler leurs orientations de travail, et les résultats qu'ils obtiennent, pour montrer la pertinence qu'ils peuvent avoir dans l'optique aujourd'hui dominante. (ii) De nouvelles pistes doivent être explorées pour répondre aux nouveaux questionnements.

Le but recherché est de maintenir en permanence un équilibre entre les sociétés humaines, qui aspirent à se développer, et les possibilités offertes par l'environnement. Cela implique une double action : (i) sur les besoins, qu'il faut modérer en décourageant l'expansion démographique, en choisissant les technologies qui économisent l'énergie et les matières rares, et en favorisant le recyclage de tous les éléments utilisés par les hommes ; (ii) sur les milieux, qu'il faudra adapter à des charges croissantes en dirigeant et en contrôlant leur fonctionnement, et en prenant en charge les opérations que la nature ne peut assumer - le traitement des eaux, par exemple, qui s'impose chaque fois que les consommations s'élèvent au-dessus d'un certain niveau, et que les processus naturels sont incapables d'éliminer certains éléments, les métaux lourds par exemple.

Les politiques de développement durable ont jusqu'ici surtout mis l'accent sur le premier volet de ces mesures. La progression rapide de l'économie de pays encore pauvres il y a une génération, comme la Chine et l'Inde, prouve qu'il est impossible d'éviter un accroissement 
des pressions sur l'environnements terrestre : cela oblige à reposer la question de l'utilisation du nucléaire ou celle de la condamnation des Organismes Génétiquement Modifiés.

Il s'agit là de questions qui impliquent la mesure de données qui ne sont pas du ressort du géographe, mais l'étude du débat, de la manière dont il est mené et de ses dimensions idéologiques est pleinement du ressort de notre discipline.

\subsection{Le choix des stratégies retenues pour assurer le développement durable est-il parfaitement satisfaisant?}

Les stratégies actuelles reposent sur l'idée que les problèmes les plus urgents à résoudre sont ceux qui résultent de déséquilibres globaux. Pour les traiter, il faut régionaliser les politiques de contrôle de la croissance et d'utilisation de l'espace et des ressources. L'ONU avait l'habitude d'aborder les problèmes du développement économique et des inégalités sociales à travers la mise en œuvre d'indicateurs. C'est la même stratégie qu'elle applique dans le domaine du développement durable. Cela explique la part prise par les économistes, les sociologues et les écologistes dans la mise au point des instruments de mesure que constituent les indicateurs. Il faut en prendre acte et comprendre comment les chercheurs des autres disciplines travaillent si l'on veut éventuellement être capable de formuler une appréciation critique de leurs méthodes.

Le recours massif aux formes concentrées d'énergie est sans doute le facteur qui contribue le plus à accroître la pression humaine sur la nature en multipliant l'émission de gaz à effet de serre. Qu'il s'agisse du problème prioritaire, nul n'en doute. Il ne faudrait cependant pas négliger les autres approches du développement durable.

Il convient de donner plus de place à l'analyse des déséquilibres d'échelle locale et régionale, et d'analyser de manière précise les milieux traditionnels, et ceux que la modernisation des genres de vie et des activités est en train de créer. C'est dans ce domaine que la contribution des géographes est sans doute la plus intéressante : comment fonctionnent les écosystèmes des espaces suburbains et rurbains ? Comment les couverts végétaux s'y transforment-ils ? Quelle conséquence cela a-t-il sur la biodiversité ?

\section{Conclusion}

Les résultats obtenus grâce à la mise en œuvre des politiques de développement durable que la Conférence de Rio de Janeiro a lancées sont considérables. Ils sont en même temps insatisfaisants : dans les pays du Sud, beaucoup de gens espéraient une lutte plus décidée contre la misère ; ceux qui mettaient surtout l'accent sur le versant social des nouvelles politiques sont déçus et militent volontiers pour l'altermondialisme - qui est la version « critique » de l'action internationale qui se développe. On l'a vu à l'occasion du Sommet sur le Développement durable, destiné à dresser le bilan d'action en ce domaine, et qui s'est tenu à Johannesburg en août 2002. Certains « verts », inspirés par Foucault, appellent à la résistance vis-à-vis de toutes les initiatives qui n'ont pas été soumises à contre-enquête et à contre-expertise (Darier, 1999, p. 235).

Les ratés de la coopération internationale sont encore dramatiques : les Etats-Unis refusent toujours de signer le protocole de Kyoto, alors qu'ils sont les premiers émetteurs de gaz à effet de serre sur la planète. 
On peut également se demander si le principe de susbtituabilité, sur lequel repose l'essentiel des politiques de développement durable actuellement engagées, ne menace pas certains des valeurs centrales de toute civilisation :

«On peut [au] sujet [des biens et des services que la nature offre aux hommes] poser les quatre questions suivantes : sont-ils techniquement substituables ? Si tel est le cas, est-il toujours économiquement opportun de substituer une procédure artificielle à un service naturel détruit ? Cette substitution est-elle esthétiquement souhaitable ? Est-elle éthiquement acceptable ? (Bourg, in Smouts, 2005, p. 23). «On voit donc que les questions soulevées par notre mode de développement actuel ne sauraient être tranchées par les seuls économistes Le développement durable est à part entière un projet de civilisation, dépassant de loin le seul prisme économique » (Bourg, in Smouts, 2005, p. 25).

L'état de la recherche scientifique ne permet pas toujours de dire dans quelle mesure la diminution de la biodiversité menace les écosystèmes :

«La grande question qu'il faut se poser est celle de savoir dans quelle mesure la perte de biodiversité est susceptible d'altérer le fonctionnement des écosystèmes, et donc indirectement les services écologiques qu'ils fournissent.

Quand on a commencé à se poser cette question il y a une dizaine d'années, on s'est aperçu qu'on ne disposait pas d'une base scientifique suffisante pour y répondre. Certes, l'importance de ce que j'appellerai la diversité "verticale" était très bien établie en écologie. [...] Ce qui était beaucoup moins clair, il y a quelques années encore, c'est l'importance de ce que j'appellerai la diversité "horizontale', c'est-à-dire la diversité des espèces au sein d'un même niveau trophique [...] » (Loreau in Smouts, 2005, p. 28-29)..

Les recherches ont heureusement beaucoup progressé en ce domaine :

«L'ensemble des résultats scientifiques récents montre que la biodiversité a un impact potentiel non négligeable sur le fonctionnement des écosystèmes naturels ou gérés par l'homme » (Loreau in Smouts, 2005, p. 29-31).

Ces limites et réserves ne doivent pas faire oublier les aspects positifs :

«Tout comme la référence à la "protection de l'environnement" dans les années soixante-dix, la référence au développement durable peut être lue comme un vecteur d'innovations politiques aussi bien en termes de défense des valeurs que de transformations des pratiques. Je développerai cet argument en deux temps. D'une part, en montrant en quoi la référence au développement durable renouvelle les choix d'action publique (policy making) par une critique de la technocratie et l'introduction de nouvelles exigences. D'autre part, en montrant comment la référence au développement durable peut conduire à poser de façon renouvelée le rapport gouvernant/gouverné » (Lascoumes, in Smouts, 2005, p. 96).

Marie-Claude Smouts éclaire de façon un peu différente ce qu'apporte l'idée de développement durable :

«Le développement durable est à la fois un outil analytique et un projet politique.

Cette double nature le place au cœur de controverses majeures. 
Outil analytique, le concept dénonce a contrario tout ce qui ne va pas dans la façon classique de considérer le développement. Mais, en désignant les conditions nécessaires d'une croissance durable, il reste dans l'option politique du développement. Projet politique, le développement durable est contestataire Il met en cause l'ordre existant. Pourquoi faudrait-il insister sur les responsabilités à l'égard des "générations futures" si le cours actuel des choses devait mener à coup sûr vers un avenir meilleur?»(Smouts, 2005, p 7-8).

\section{Références}

Allègre, Claude, 1990, Economiser la Planète, Paris, Fayard, 381 p.

Aubertin, Catherine, Hourcade, Jean-Charles, Vien, Jean-Dominique, 2006, in Aubertin, Catherine, Vivien, Franck-Dominique (eds.), Le Développement durable. Enjeux politiques, économiques et sociaux, op. cit., p. 49-74.

Aubertin, Catherine, Vivien, Franck-Dominique (eds.), 2006, Le Développement durable. Enjeux politiques, économiques et sociaux, Paris, la Documentation française, 143 p.

Barbault, Robert,1997, Biodiversité, Paris, Hachette, 159 p.

Blaikie, Piers, Brookfield, Harold, 1987, Land Degradation and Society, Londres, Methuen.

Blondel, J., 2005, « Biodiversité et sciences de la nature », in : Marty, P., Vivien, F.-D, Lepart, J., Larrère, R. (eds.), Les Biodiversités : objets, théories, pratiques, Paris, Ed. du CNRS, p. 23-36.

Boisvert, Valérie, Vivien, Jean-Dominique, 2006, «Le développement durable : une histoire de controverses économiques », in Aubertin, Catherine, Vivien, Franck-Dominique (eds.), Le Développement durable. Enjeux politiques, économiques et sociaux, op. cit., p. 15-48.

Bourg, Dominique, 1993, Les Sentiments de la nature, Paris, la Découverte.

Bourg, Dominique, 1996, Les Scénarios de l'écologie. Débat avec Jean-Paul Deléage, Paris, Hachette, 142 p.

Brunel, Sylvie, 2005, « La Terre plutôt que les Terriens ? Développement du Sud et développement durable », in Gauchon, Pascal, Tellenne, Cédric (eds.), Géopolitique du développement durable, op. cit., p. 109-132.

Brunel, Sylvie, 2005, « Priorité aux hommes ! Le développement durable en Afrique », in Gauchon, Pascal, Tellenne, Cédric (eds.), Géopolitique du développement durable, op. cit., p. 335-352.

Buttimer, Anne (ed.), 2001, Sustainable Landscapes and Lifeways. Scale and Appropriateness, Cork, University of Cork Press, XXII-398 p.

Buttimer, Anne, Stol, Taeke, 2001, «Flows of food and fuel in Germany, Ireland, the Netherlands and Sweden, 1960-1990 », in Buttimer, Anne (ed.), Sustainable Landscapes and Lifeways. Scale and Appropriateness, op. cit., p. 79-103.

Cadoret, A. (ed..), 1985, Protection de la nature. Histoire et idéologie. De la nature à l'environnement, Paris, l'Harmattan.

Chartier, Denis, Ollitraut, Sylvie, 2006, «ONG et développement durable : les liaisons dangereuses », in Aubertin, Catherine, Vivien, Franck-Dominique (eds.), Le Développement durable. Enjeux politiques, économiques et sociaux, op. cit., p. 94-114.

Claval, Paul, 2000, «Ethique et nature. Une approche conceptuelle », Géographie et cultures, n 37, p. 3-22.

Claval, Paul, 2001, « The Geographical Study of Myths », Norsk Geogrfisk Tidsskrift, vol. 55, n 3, p. 138-151.

CMEE (Commission mondiale sur l'Environnement et le Développement), 1988, Notre Avenir à tous, Montréal, Editions du Fleuve. 37, p. 4-22. 
Coase, Ronald H., 1960, « The problem of social cost », Journal of Law and Economics, $\mathrm{n}^{\circ}$ 3, p. 1-44.

Cobast, Eric, 2005, «L'insoutenable responsabilité des hommes - Le regard du philosophe », in : Gauchon, Pascal, Tellenne, Cédric (eds.), Géopolitique du développement durable, op. cit., p. 23-29.

Costanza, Robert, Perrings, Charles, Cleveland, Cutler J. (eds), 1997, The Development of Ecological Economics, Cheltenham, Edward Elgar.

Dales, John, 1968, Pollution, Property and Prices. An Essay in Policy Making and Economics, Toronto, Toronto University Press.

Daly, H. E., 1990, « Toward some Operational Principles of Sustainable Development », Ecological Economics, vol. 2, $\mathrm{n}^{\circ}$ 1, April,p. 1-6.

Darier, Eric (ed), 1999, Discourses of the Environment, Oxford, Blackwell, XII-275 p.

Deléage, Jean-Paul, 1991, Histoire de l'écologie. Une science de l'homme et de la nature, Paris, la Découverte, $330 \mathrm{p}$.

Domar, Eusey D., 1946, «Capital Expansion, Rate of Growth and Employment », Economietrica, avril 1946 ; repris, dans Domar, Eusey D., 1957, Essays in the Theory of Economic Growth, New York, Oxford University Press.

Dorst, Jean, 1965, Avant que Nature ne meure, Neuchâtel, Delachaux et Niestlé.

Ducroux, Anne-Marie (ed.), 2002, Les Nouveaux Utopistes du développement durable, Paris, Autrement.

Ekman, Soren, 1998, Vers une Ecologie industrielle. Comment mettre en pratique le développement durable dans une société hyper-industrielle, Paris, Charles Léopold Meyer.

Ferrandéry, Jean-Luc, 2005, «De l'aménagement au ménagement du territoire : aménagement du territoire et développement durable », in Gauchon, Pascal, Tellenne, Cédric (eds.), Géopolitique du développement durable, op. cit., p. 181-199.

Ferry, Luc, 1992, Le Nouvel Ordre écologique. L'arbre, l'animal et l'homme, Paris, Grasset, 275 p.

Gauchon, Pascal, Tellenne, Cédric (eds.), 2005, Géopolitique du développement durable, Paris, Presses Universitaires de France, 365 p.

Georgescu-Rœgen, Nicholas, 1995, La Décroissance : entropie-écologie-économie, Paris, Sang de la Terre.

Grinevald, Jacques, 2002, «L'économie de la décroissance », L'Ecologiste, n 8, p 69-70.

Grossman, Gene M., Krueger, Alan B., 1995, « Economic growth and the environment », The Quaterly Journal of Economics, $\mathrm{n}^{\circ} 2$, p.. 353-377.

Guillaud, Yann, 2005, Diversité biologique et développement durable, Paris, Karthala-Unesco.

Hägerstrand, Torsten, 2001, «A look at the political geography of environmental management », in Buttimer, Anne (ed.), Sustainable Landscapes and Lifeways. Scale and Appropirateness, op. cit., p. 35-58.

Hardin, Garrett, 1968, « The tragedy of the commons », Science, vol. 162, n 3859, dec., p. 1343-1348.

Harribey, Jean-Marie, 1997, L’Economie économique Le développement soutenable par la réduction du temps de travail, Paris, 1'Harmattan.

Harrod, Roy F., 1939, « Essays in Dynamic Theory », Economic Journal, mars.

Hartwick, John M, 1977, «Intergenerational Equity in the Investing of Rents from Exhaustible Resources », The American Economic Review, vol. 67, n 5, dec., p. 972-974.

Hawken, Paul, Lovins, Armory, Lovins, L Hunter, 2000, Natural Capitalism. Creating the Next Industrial Revolution, New York, First Black Bay Books.

Hayward, Tim, 1994, Ecological Thought. An Introduction, Cambridge, Polity Press, XII-259 p.

Hervieu-Léger, D., Hervieu, B., 1979, Le Retour de la nature : au fond de la forêt, l'Etat, Paris, le Seuil, 234 p.

Hunt, Diana, 1989, Theories of Economic Development, Londres, Harvester Wheastsheaf.

Huston, M A., 1994, Biodiversity. The Coexistence of Species on Changing Landscape, Cambridge, Cambridge University Press, $681 \mathrm{p}$. 
ICC, 1991, The Business Charter for Sustainable Development. Principles for Environmental Management, Paris, International Chamber of Commerce.

Illich, Ivan, 1973, La Convivialité, Paris, le Seuil.

Jonas, Hans, 1990, Le Principe Responsabilité. Une éthique pour la civilisation technologique, Paris, le Cerf.

Keynes, John M., 1936, 'The General Theory of Employment, Interest and Money, Londres, Macmillan.

Larrère C. et Larrère R. (eds.), 1997, La Crise environnementale, Paris, INRA-Editions.

Larrère, R., 2005, «Quand l'écologie, science de l'observation, devient science de l'action. Remarques sur le génie écologique », in : Marty, P., Vivien, F.-D, Lepart, J., Larrère, R. (eds.), Les Biodiversités : objets, théories, pratiques, Paris, Ed. du CNRS, p. 173-194.

Lascoumes, Pierre, 1994, L'Ecopouvoir. Environnement et politiques, Paris, la Découverte.

Lefèvre, Mathias, 2006, «La mobilisation du monde des affaires : formes et significations », in Aubertin, Catherine, Vivien, Franck-Dominique (eds.), Le Développement durable. Enjeux politiques, économiques et sociaux, op. cit., p. 115-134.

Lenglet, Stéphane, 2005, «Le salut par l'Etat ou par le marché ? L'Etat et le développement durable », in Gauchon, Pascal, Tellenne, Cédric (eds.), Géopolitique du développement durable, op. cit., p. 213-236.

Lepart, J., 1997, « La crise environnementale et les théories de l'équilibre en écologie », in Larrère C. et Larrère R. (eds.), La Crise environnementale, op. cit., p. 131-144.

Le Prestre, Philippe, 1997, Ecopolitique internationale, Montréal, Guérin Universitaire.

Le Prestre, Philippe (ed.), 2002, Governing Global Biodiversity. The Evolution and Implementation of the Convention on Biological Diversity, Ashgate, Aldershot.

Le Treut, Hervé, Janovici, Jean-Marc, 2004, L’Effet de serre. Allons-nous changer le climat? Paris, Flammarion, 2ème éd.

Maréchal, Jean-Paul, Quenault, Béatrice (eds.), 2005, Le Développement durable. Une perspective pour le XXI siècle, Rennes, Presses Universitaires de Rennes.

Martin, Jean-Yves, 2006, «La France et le développement durable », in Aubertin, Catherine, Vivien, FranckDominique (eds.), Le Développement durable. Enjeux politiques, économiques et sociaux, op. cit., p. 75-91.

Martinez-Alier, Joan, 2002, The Environmentalism of the Poor, Cheltenham, Edward Elgar

Marty, P., Vivien, F.-D, Lepart, J., Larrère, R. (eds.), 2005, Les Biodiversités : objets, théories, pratiques, Paris, Ed. du CNRS.

Mayeur, Arnaud, 2005, « Le regard de l'économiste », in Gauchon, Pascal, Tellenne, Cédric (eds.), Géopolitique du développement durable, op. cit., p. 69-92.

McComich, John, 1995, The Global Environmental Movement, Chichester, John Wiley.

Meadows, Donella H., Meadows, Denis L, Randers, Jorgen, Behrens, W.W. III, 1972, Halte à la croissance, Paris, Fayard.

Milton, Kay, 1996, Environmentalism and Cultural Theory. Exploring the Role of Anthropology in Environmental Discourses, Londres, Routledge, IX-266 p

Myers, N., Mittermeier, R. A., Mittermeier, C. G., da Fonseca, G. A. et Kent J., 2000, « Biodiversity hotspots for conservation priorities », Nature, $\mathrm{n}^{\circ} 403$, p. 853-858.

Passet, René, 1996, L'Economique et le vivant, Paris, Payot.

Pepper, David, 1996, Modern Environmentalism. An Introduction, Londres, Routledge, VIII-376 p

Pickett, S. T., White, P. S., 1985, The Ecology of Natural Disturbance and Patch Dynamics, New York, Academic Press, 492 p.

Picon, B., 1988, L'Espace et le temps en Camargue, Arles, Actes-Sud, 234 p.

Proglio, Henri, 2005, «L'entreprise responsable », in Gauchon, Pascal, Tellenne, Cédric (eds.), Géopolitique du développement durable, op. cit., p. 255-259. 
Ramade, François, 1999, Le Grand Massacre. L'avenir des espèces vivantes, Paris, Hachette.

Rifkin, Jeremy, 1998, Le Siècle biotech, Paris, la Découverte.

Rist, Gilbert, 1996, Le Développement durable. Histoire d'une croyance occidentale, Paris, Presses de Scoiences Politiques.

Riutort, Philippe, 2005, « Métamorphose d'un phénomène public - Le regard du sociologue », in Gauchon, Pascal, Tellenne, Cédric (eds.), Géopolitique du développement durable, op. cit., p. 94-105.

Robbins, Paul, 2004, Political Ecology : a Critical Introduction, Oxford et Malden (Mass.), Blackwell, XXI-240 p. Rostow, W. W., 1960, The Stages of Economic Growth, Cambridge, Cambridge University Press.

Rougerie, Gabriel, Beroutchachvili Nicolas, 1991, Géosystèmes et paysages. Bilan et méthodes, Paris, Armand Colin. Sachs, Ignaszy, 1980, Stratégies de l'écodéveloppement, Paris, Economie et Humanisme et Editions ouvrières.

Sacquet, Anne-Marie, 2002, «Donner corps aux principes », in Ducroux, Anne-Marie (ed.), Les Nouveaux Utopistes du développement durable, op. cit., p. 53-58.

Saint-Marc, Philippe, 1994, L'Economie barbare, Paris, Editions Frison-Roche, 491 p.

Smouts, Maris-Claude (ed.), 2005, Le Développement durable. Les termes du débat, Paris, Armand Colin, 2005, 289 p.

Solow, Robert M., 1972, Théorie de la croissance économique, Paris, Payot.

Solow Robert M., 1977, « The Economics of Resources, or the Resources of Economics », The American Economic Review, vol. 64, n² 2, p. 1-14.

Solow, Robert M., 1999, «An Almost Practical Step toward Sustainability », in Oates, Wallace E., (ed), The RFF Reader in Environmental Resource Management, Washington, D. C., Resources for the Future.

Soyez, Dietrich, 2001, « "Bottom-up” globalization : trans-national lobbies and industrial change », in Buttimer, Anne (ed.), Sustainable Landscapes and Lifeways. Scale and Appropirateness, op. cit., p. 59-78.

Tellenne, Cédric, 2005, « Solidaires, vraiment. La "société civile" mondiale et le développement durable », in Gauchon, Pascal, Tellenne, Cédric (eds.), Géopolitique du développement durable, op. cit., p. 261-276.

UICN, PNUE et WWF, 1980, Stratégie mondiale de la croissance La conservation des espèces vivantes au service du développement durable, Gland, UICN/PNUE/WWF.

Vivien, F. D., 2005, « La diversité biologique entre valeurs, évaluations et valorisations économiques », in : Marty, P., Vivien, F.-D, Lepart, J., Larrère, R. (eds.), Les Biodiversités : objets, théories, pratiques, Paris, Ed. du CNRS, p. 125-140.

Voisenet, Jacques, 2005, «De l'écologie au développement durable », in Gauchon, Pascal, Tellenne, Cédric (eds.), Géopolitique du développement durable, op. cit., p. 7-22.

WBCSC, 2001, Sustainability through the Market : Seven Keys to Success, Genève, World Business District for Sustainable Development. 
